\title{
Progesterone alters Nogo-A, GFAP and GAP-43 expression in a rat model of traumatic brain injury
}

\author{
FENG LIU $^{1}$, FAN LIAO $^{1}$, WEI LI ${ }^{2}$, YONGFENG HAN ${ }^{3}$ and DAGUANG LIAO ${ }^{1}$ \\ ${ }^{1}$ Department of Neurosurgery, The Third Xiangya Hospital of Central South University; \\ ${ }^{2}$ Department of Neurosurgery, The First Hospital of Changsha, Changsha, Hunan 410013; \\ ${ }^{3}$ The First Hospital of Shijiazhuang City, Shijiazhuang, Hebei 050000, P.R. China
}

Received June 28, 2013; Accepted January 31, 2014

DOI: $10.3892 / \mathrm{mmr} .2014 .1967$

\begin{abstract}
Previous studies have demonstrated that progesterone has neuroprotective effects in the central nervous system (CNS) following traumatic brain injury (TBI). Numerous cellular mechanisms have been reported to be important in the neuroprotective effects of progesterone, including the reduction of edema, inflammation and apoptosis, and the inhibition of oxidative stress. However, the effect of progesterone on neuronal protection following TBI remains unclear. The present study aimed to investigate the effects of progesterone on the expression of Nogo-A, an inhibitor of axonal growth, glial fibrillary acidic protein (GFAP), a main component of the glial scar and growth-associated protein-43 (GAP-43), a signaling molecule in neuronal growth in TBI rats. The TBI model was produced by the weight drop method. In total, 75 rats were assigned to three groups: the sham group, TBI group with vehicle treatment and TBI group with progesterone treatment. The protein expression of Nogo-A, GFAP and GAP-43 in the cortex and the hippocampus was examined by immunocytochemistry. TBI rats significantly increased the expression of Nogo-A, GFAP, and GAP-43 at 1, 3, 7 and 14 days postinjury. Progesterone significantly decreased the expression of Nogo-A and GFAP, and upregulated the GAP-43 protein. Our findings suggested that progesterone promotes neuroprotection following TBI by inhibiting the expression of Nogo-A and GFAP, and increasing GAP-43 expression.
\end{abstract}

\section{Introduction}

Traumatic brain injury (TBI) is the leading cause of injury-related mortality and disability among young people worldwide (1). Although more individuals survive from TBI now than in the

Correspondence to: Dr Daguang Liao, Department of Neurosurgery, The Third Xiangya Hospital of Central South University, 138 Tongzipo Road, Changsha, Hunan 410013, P.R. China E-mail: daguangliao@hotmail.com

Key words: progesterone, traumatic brain injury, Nogo-A, GFAP, GAP-43 past, survivors often suffer residual physical, emotional, behavioral and cognitive impairments, resulting from TBI-induced pathological lesions in the brain. Although substantial efforts have been made to develop a new treatment for TBI, the treatment has been hampered by a lack of understanding of the molecular and cellular mechanisms underlying TBI.

Recently, several studies have demonstrated the neuroprotective effect of progesterone in the CNS following TBI $(2,3)$. However, the molecular mechanisms underlying the neuroprotective effect of progesterone following TBI remains unclear. It has been reported that progesterone improves cognition and movement function, decreases cerebral edema, inhibits inflammation and reduces apoptosis (2-6). However, the effect of progesterone on neuronal regeneration and plasticity following TBI has not yet been well studied.

It is well known that damage to the central nervous system (CNS) following TBI is detrimental due to the inability of central neurons to regenerate their axons and dendrites (7). The failure of the CNS to regenerate is not due to the intrinsic deficit of regenerative capabilities, but is caused by the damaged environment that either does not support or inhibits neuronal regeneration (7). Several inhibitors of axonal growth have been identified in myelin, including Nogo-A (8), myelin-associated glycoprotein (9) and oligodendrocyte myelin glycoprotein (10). Nogo-A, which is highly expressed in oligodendrocytes, is considered to be the most important inhibitor of axonal growth in models of CNS injury (7). In addition, the failure of axonal regeneration may also result from the presence of a glial scar, which prevents neuronal regrowth $(11,12)$. Glial fibrillary acidic protein (GFAP), specifically expressed in astrocytes, forms a main component of the glial scar (12). Increased Nogo-A and GFAP expression has been reported in experimental injury to the peripheral nervous system and CNS in rodents (13-15). However, the effect of progesterone on the expression of Nogo-A and GFAP following TBI has yet to be examined.

Growth-associated protein-43 (GAP-43), expressed in the neuronal growth cone, is involved in the transduction of intracellular and extracellular signals that regulate neuron growth, synaptic formation and synaptic plasticity (16-18). GAP-43 is greatly upregulated during brain development, at the time of neurite outgrowth and during synaptogenesis $(16,18)$. Several lines of evidence demonstrated that GAP-43 is upregulated in regeneration and plasticity 
following TBI in rat models (19-22). Therefore, it is noteworthy to examine whether progesterone increases GAP-43 expression following TBI.

Although progesterone has been reported to promote remyelination and axonal regeneration in experimental models of spinal neurodegeneration and peripheral nerve injury (23-25), the roles of progesterone in axonal regeneration in the brain following injury remain unknown. In the present study, the expression of Nogo-A, GFAP and GAP-43 in TBI rats were investigated and the effects of progesterone on their expression at $1,3,7,14$ and 28 days post-injury was examined. The present study revealed that progesterone significantly decreased the expression of Nogo-A and GFAP, and upregulated the GAP-43 protein, suggesting that progesterone promoted neuronal regeneration and plasticity in TBI rats.

\section{Materials and methods}

Animals and the TBI model. Animal experimental protocols were approved by the Committee for Animal Experiments at The Third Xiangya Hospital of Central South University (Changsha, China). In total, 75 adult male Sprague Dawley rats (weighing 250-300 g) were housed at a constant temperature $\left(23^{\circ} \mathrm{C}\right)$ and humidity $(7 \%)$ with a $12 \mathrm{~h} \mathrm{light} / \mathrm{dark}$ cycle, and maintained on standard pelleted rat chow and water ad libitum. They were habituated to the housing conditions for at least a week prior to the surgical procedure. The TBI model was produced by the weight drop method as previously described (26). Briefly, rats were intraperitoneally anesthetized with $10 \%$ chloral hydrate $(3 \mathrm{mg} / \mathrm{kg})$ and were placed in a stereotaxic frame. During the surgical procedure, body temperature was kept at $37^{\circ} \mathrm{C}$. After the skull was exposed, a $5 \mathrm{~mm}$ craniotomy was performed over the right frontoparietal cortex to expose the dura, with the center of the opening located $2 \mathrm{~mm}$ lateral to the midline and $2.2 \mathrm{~mm}$ posterior to the bregma. A $20 \mathrm{~g}$ weight was dropped from a height of $30 \mathrm{~cm}$ onto a piston resting on the exposed dura, resulting in a moderate injury in the right brain. The rats that experienced brief convulsion and apnea immediately following the TBI were included in the present study and the rats that died or did not exhibit consciousness loss and neurological dysfunction following recovery were excluded. The locomotor behavior of rats following TBI was assessed, including balancing (5 points), righting (1 point), head-holding (1 point), walking (4 points), escaping behavior in response to tail clamping ( 2 points) and paw withdrawal in response to tail clamping ( 2 points). Rats with a total of 10 or above $(n=2)$ were excluded from the present study. In the sham rats, the same surgical procedure was performed, however, no weight was dropped on the dura.

The rats were assigned to three groups: a sham group with no progesterone treatment $(\mathrm{n}=25)$, a TBI group with vehicle treatment $(n=25)$ and a TBI group with progesterone treatment $(\mathrm{n}=25)$. In each group, the rats were sacrificed at 1 $(\mathrm{n}=5), 3(\mathrm{n}=5), 7(\mathrm{n}=5), 14(\mathrm{n}=5)$ and 28 days $(\mathrm{n}=5)$ following surgery. Progesterone (10 mg/kg, qd) or 2-hydroxypropyl$\beta$-cyclodextrin (as the vehicle control) was injected intraperitoneally $6 \mathrm{~h}$ after the surgery and continued until the rats were sacrificed. The dose and duration of progesterone used in the present study was similar to that used in previous studies $(27,28)$.
Tissue preparation. At specific time points $(1,3,7,14$ and 28 days after surgery), rats were intraperitoneally anesthetized with $10 \%$ chloral hydrate $(3 \mathrm{mg} / \mathrm{kg})$. The rats were then perfused via the left ventricle with cold phosphate-buffered saline, followed by $4 \%$ paraformaldehyde. The brains were dissected and post-fixed with $4 \%$ formaldehyde at $4{ }^{\circ} \mathrm{C}$ overnight. The tissues were then dehydrated with ethanol, cleared in xylene, filtrated in paraffin wax and embedded in paraffin wax. Serial sections ( $4 \mu \mathrm{m}$ thick) were obtained using a microtome (Leica, Bensheim, Germany).

Immunocytochemistry. Tissue sections ( $4 \mu \mathrm{m}$ thick) were obtained from paraffin-embedded tissue blocks. The tissue sections were immunocytochemically stained for Nogo-A, GFAP and GAP-43. Briefly, sections were washed in xylene to remove the paraffin, rehydrated with serial dilutions of alcohol, followed by washing in a PBS solution. The samples were then incubated in primary antibodies against Nogo-A (1:200 dilution), GFAP (1:200 dilution) and GAP-43 (1:200 dilution), or non-immune rabbit $\mathrm{IgG}$ at $4^{\circ} \mathrm{C}$ overnight. Non-immune rabbit $\operatorname{IgG}$ was used as the negative control to rule out the non-specific staining (Fig. 1). All primary antibodies and the non-immune rabbit IgG were rabbit anti-rat antibodies, purchased from Beijing Biosythesis Biotechnology Co., Ltd. (Beijing, China). Following primary antibodies being washed off, sections were incubated with goat anti-rabbit biotin-conjugated secondary antibodies (1:1,000 dilution; Beijing Biosynthesis Biotechnology Co., Ltd) for $20 \mathrm{~min}$ at $37^{\circ} \mathrm{C}$. The tissue sections were then incubated with streptavidin horseradish peroxidase for $20 \mathrm{~min}$ at $37^{\circ} \mathrm{C}$. The DAB substrate was applied to the section for $2 \mathrm{~min}$ and then the sections were examined under a light microscope.

Coronal brain sections were selected between 3 and $4 \mathrm{~mm}$ posterior to the bregma (starting $\sim 1 \mathrm{~mm}$ posterior to the lesion) at the level of the cortex and the hippocampus. Images were captured at the same magnification (x200) by a digital camera (Nikon, Tokyo, Japan). Four sections at the same location from each rat were selected to compare and five random fields without overlaps in each section were evaluated. The average numbers of positive cells were used for analysis.

Statistical analysis. Analyses were performed using SPSS 13.0. All values are presented as the mean \pm standard deviation. One-way analysis of variance (ANOVA) was used to compare the differences among the control, the TBI group with vehicle treatment and the TBI group with progesterone treatment, followed by a post-hoc LSD test. $\mathrm{P}<0.05$ was considered to indicate a statistically significant difference.

\section{Results}

Progesterone decreases Nogo-A expression in TBI rats. The protein expression of Nogo-A was detected in the cortex $1 \mathrm{~mm}$ posterior to the lesions from the sham rats, TBI rats treated with the vehicle control and TBI rats treated with progesterone using immunocytochemistry (Fig. 2). Positive Nogo-A immunoreactivity was observed in the oligodendrocytes. The number of positive immunoreactive cells at 1, 3, 7, 14 and 28 days after TBI was analyzed. In the sham group, only a few positive Nogo-A immunoreactive cells were identified (Fig. 2K). 

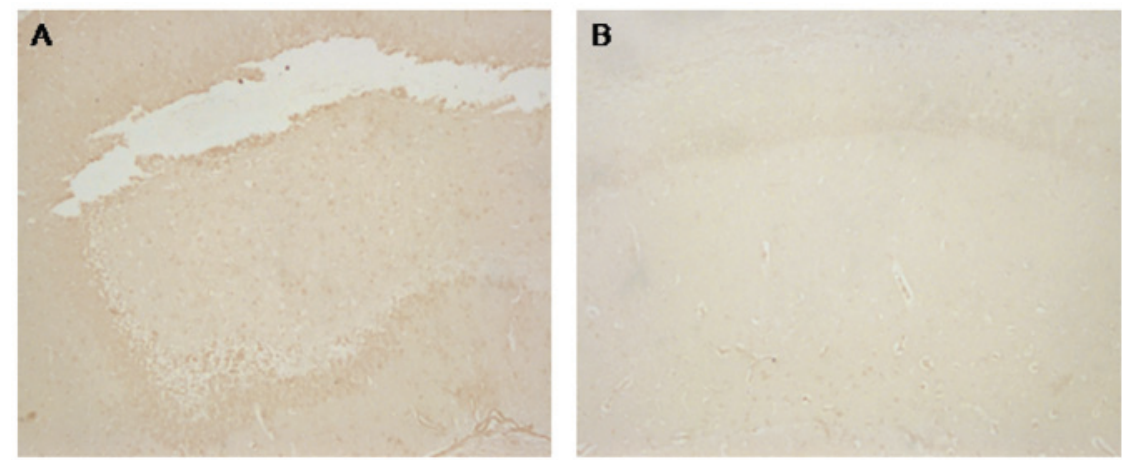

Figure 1. Photomicrographs of non-immune IgG immunostaining in the cortex (A) hippocampal region and (B) adjacent to the lesions from TBI rats. TBI, traumatic brain injury.

TBI

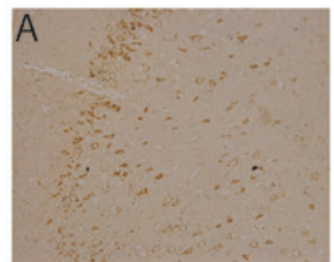

TBI

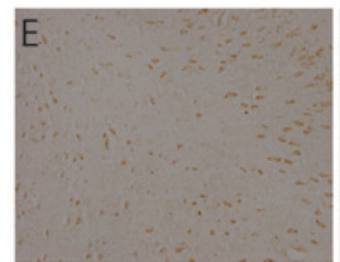

$\mathrm{TBI}$

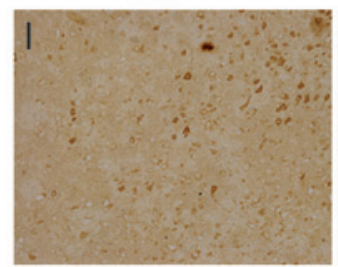

TBI+Pro

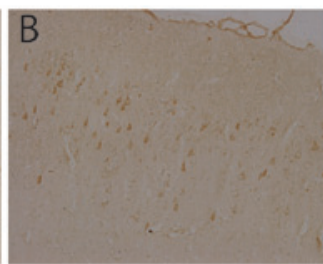

$\mathrm{TBI}+$ Pro

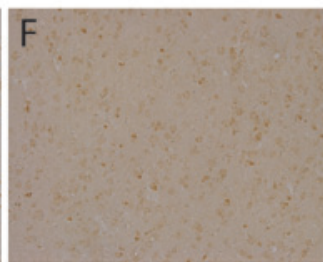

TBI+Pro

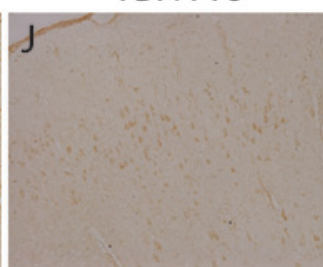

TBI

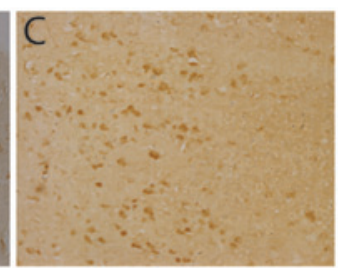

$\mathrm{TBI}$

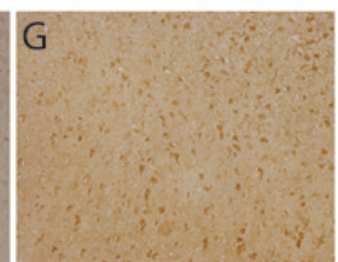

control

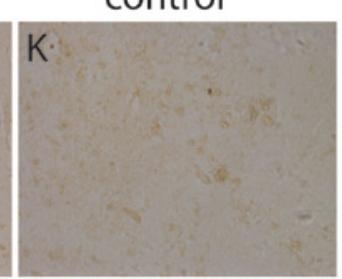

$\mathrm{TBI}+$ Pro

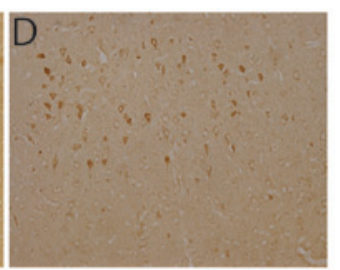

$\mathrm{TBI}+$ Pro

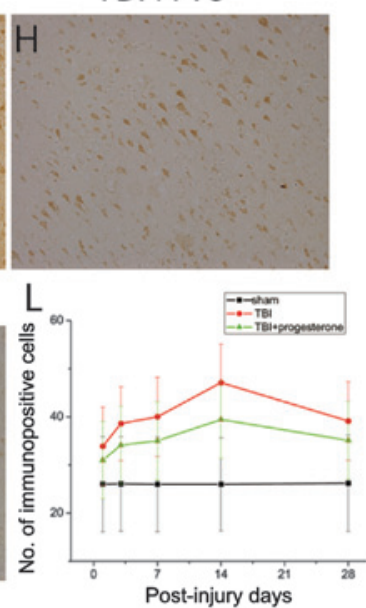

Figure 2. Photomicrographs of Nogo-A immunostaining in the cortex $1 \mathrm{~mm}$ posterior to the lesions from TBI rats treated with (A, C, E, G, I) the vehicle control and (B, D, F, H, J) progesterone at (A and B) 1, (C and D) 3, (E and F) 7, (G and H) 14 and (I and J) 28 days post-injury; (K) sham rats. (L) Number of immunopositive cells in the sham rats, TBI rats treated with the vehicle control and TBI rats treated with progesterone at $1,3,7,14$ and 28 days post-injury. TBI, traumatic brain injury.

However, compared with the sham rats, TBI rats significantly increased the Nogo-A expression at $1,3,7,14$ and 28 days postinjury (P<0.05; Fig. 2A, C, E, G, I and L). The time course of Nogo-A expression is shown in Fig. 3L. The Nogo-A expression increased on day 1 , reached a peak on day 14 and declined to a level above the control 28 days post-injury. Compared with the vehicle control, progesterone significantly decreased the expression of Nogo-A in TBI rats 1, 3, 7, 14 and 28 days postinjury ( $\mathrm{P}<0.05$; Fig. 2B, D, F, H, J and L).

Progesterone downregulates GFAP expression in TBI rats. The protein expression of GFAP was then examined in the hippocampal CA1 region from sham-injured rats, TBI rats treated with the vehicle control and TBI rats treated with progesterone, using immunocytochemistry (Fig. 3). Positive
GFAP immunoreactivity was observed in the astrocytes at 1,3, 7, 14 and 28 days in the sham rats (Fig. 3K and L). In TBI rats, increased GFAP expression was observed in the CA1 region. In TBI rats, GFAP expression was significantly increased at $1,3,7,14$ and 28 days post-injury compared with that in the sham rats $(\mathrm{P}<0.05$; Fig. $3 \mathrm{~A}, \mathrm{C}, \mathrm{E}, \mathrm{G}, \mathrm{I}$ and $\mathrm{L})$. The GFAP protein expression increased on day 1 , reached a peak on day 3 and gradually declined to a control level 28 days post-injury. Compared with the vehicle control, progesterone significantly decreased the expression of GFAP in TBI rats 3, 7 and 14 days post-injury ( $\mathrm{P}<0.05$; Fig. 3B, D, F, H, J and L).

Progesterone increases GAP-43 expression in TBI rats. The present study also investigated the GAP-43 expression in the cortex $1 \mathrm{~mm}$ posterior to the lesions from sham rats, 


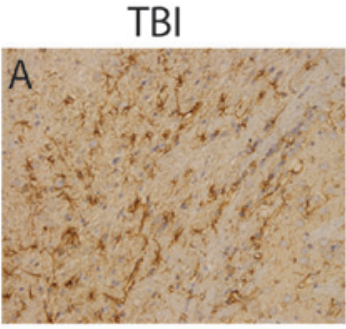

$\mathrm{TBI}$

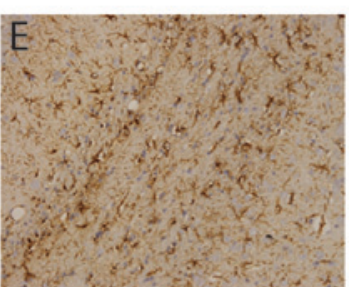

$\mathrm{TBI}$

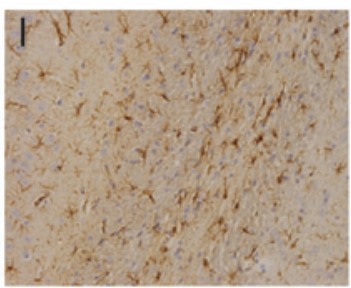

TBI+Pro

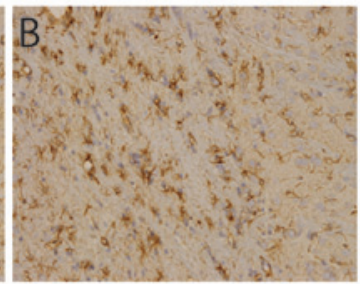

$\mathrm{TBI}+$ Pro

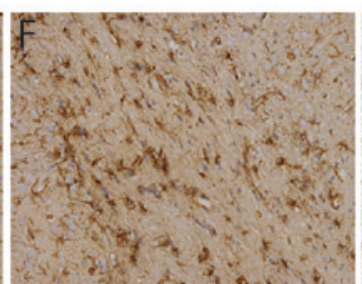

$\mathrm{TBI}+$ Pro

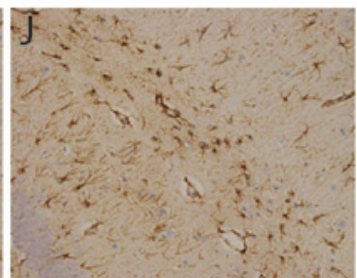

$\mathrm{TBI}$

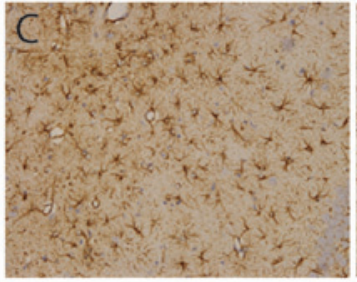

TBI

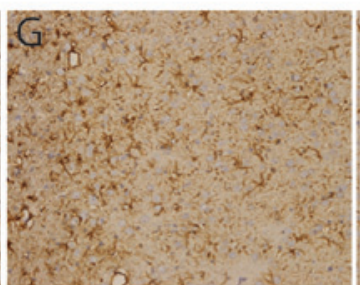

control

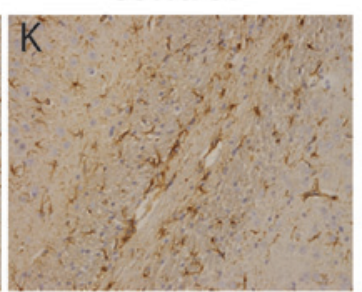

$\mathrm{TBI}+\operatorname{Pro}$

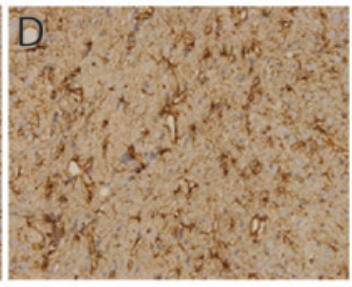

$\mathrm{TBI}+\operatorname{Pro}$
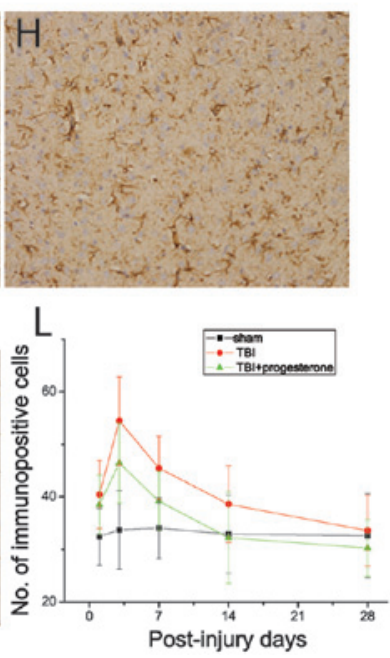

Figure 3. Photomicrographs of GFAP immunostaining in hippocampal CA1 regions from TBI rats treated with (A, C, E, G, I) the vehicle control and (B, D, F, H, J) progesterone at (A and B) 1, (C and D) 3, (E and F) 7, (G and H) 14 and (I and J) 28 days post-injury; (K) sham rats. (L) Number of immunopositive cells in the sham rats, TBI rats treated with the vehicle control and TBI rats treated with progesterone at $1,3,7,14$ and 28 days post-injury. TBI, traumatic brain injury; GFAP, glial fibrillary acidic protein.

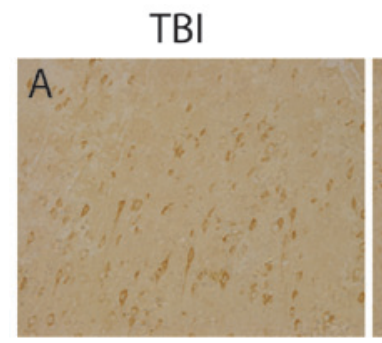

TBI

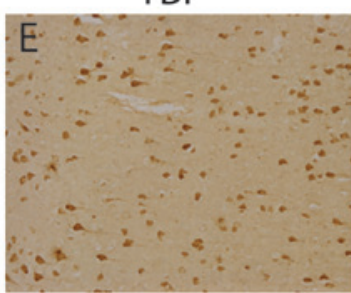

TBI

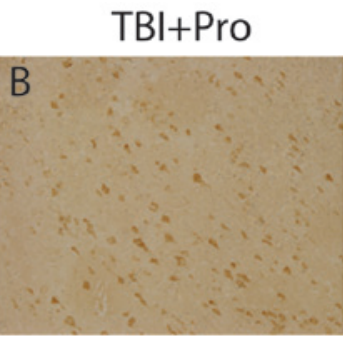

$\mathrm{TBI}+\operatorname{Pro}$

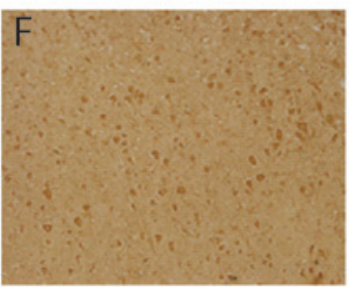

TBI+Pro

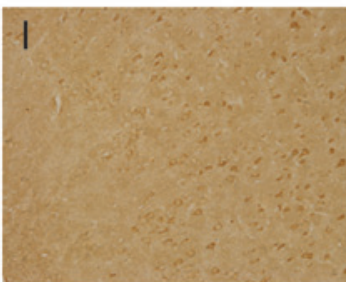

$\mathrm{TBI}$

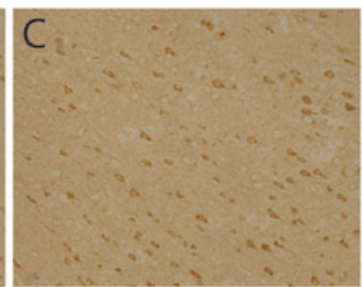

TBI
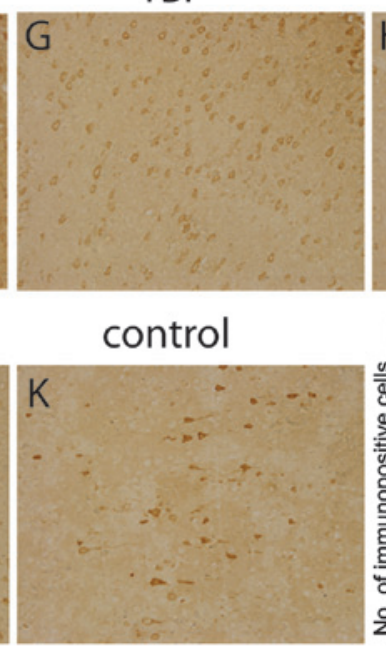

TBI+Pro

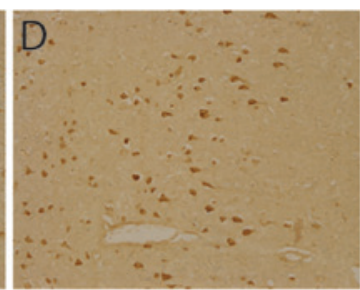

$\mathrm{TBI}+$ Pro
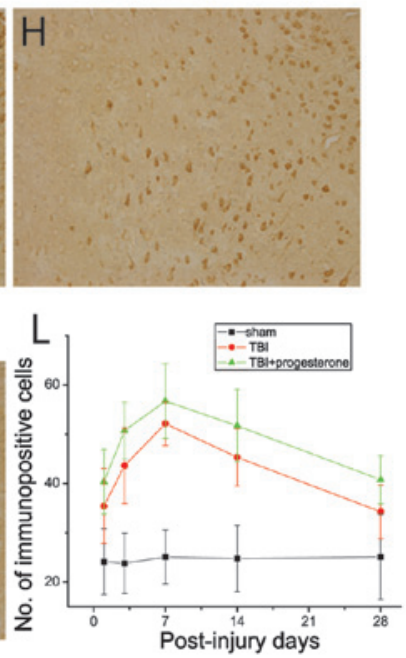

Figure 4. Photomicrographs of GAP-43 immunostaining in the cortex from TBI rats treated with (A, C, E, G, I) the vehicle control and (B, D, F, H, J) progesterone at (A and B) 1, (C and D) 3, (E and F) 7, (G and H) 14 and (I and J) 28 days post-injury; (K) sham rats. (L) Number of immunopositive cells in the sham rats, TBI rats treated with the vehicle control and TBI rats treated with progesterone at 1, 3, 7, 14 and 28 post-injury. TBI, traumatic brain injury; GAP-43, growth-associated protein- 43 . 
TBI rats treated with the vehicle control and TBI rats treated with progesterone using immunocytochemistry (Fig. 4). In the sham rats, GAP-43 positive immunoreactivity was identified in the perinuclear cytoplasm of neurons in the cortex. In TBI rats, GAP-43 expression was significantly increased at 1, 3, 7 and 14 days post-injury compared with that in the sham rats ( $\mathrm{P}<0.05$; Fig. 4A, C, E, G, I and L). The GAP-43 protein expression increased on day 1 , reached a peak on day 7 and gradually declined to a level above the control 28 days post-injury. Progesterone significantly increased the expression of GAP-43 in TBI rats at 1, 3, 7, 14 and 28 days post-injury, compared with the vehicle control ( $\mathrm{P}<0.05$; Fig. 4B, D, F, H, J and L).

\section{Discussion}

The neuroprotective role of progesterone has been established in the experimental models of TBI $(2,3)$. However, the mechanisms underlying these neuroprotective effects have not been well characterized. Numerous cellular mechanisms have been reported to be important in the neuroprotective effects of progesterone, including the reduction of edema, inflammation and apoptosis, and the inhibition of oxidative stress $(2-6,29,30)$. However, it is unclear whether progesterone can improve neuronal regeneration following TBI. One of the therapeutic goals in TBI is to identify ways of repairing and regenerating the damaged or lost neural circuit. The present study revealed that progesterone decreases the protein expression of Nogo-A, an inhibitor of axonal growth, downregulates the expression of GFAP, the main component of the glial scar and enhances the expression of GAP-43, a signaling molecule involved in neuronal growth and synaptic formation. Our findings demonstrated that progesterone is able to improve the regeneration of damaged neurons following TBI. Since Nogo-A, GFAP and GAP-43 are known to be involved in neuroregeneration $(7,11,12,18,20,21)$, our data suggest that progesterone may increase neuroprotection following TBI by inhibiting the expression of Nogo-A and GFAP and increasing the expression of GAP-43.

The dose-response study of progesterone on the behavioral performance of rats following cortical injury revealed that 8 and $16 \mathrm{mg} / \mathrm{kg}$ doses of progesterone was better than $32 \mathrm{mg} / \mathrm{kg}$ administered at 1,6 and $24 \mathrm{~h}$ with repeated administration every $24 \mathrm{~h}(27)$, suggesting that $8-16 \mathrm{mg} / \mathrm{kg}$ is an optimal dose of progesterone in the treatment of TBI. In the present study, a $10 \mathrm{mg} / \mathrm{kg}$ dose of progesterone was intraperitoneally administered $6 \mathrm{~h}$ after TBI until the rats were sacrificed. This protocol is similar to a study by Anderson et al whereby progesterone was administered intraperitoneally every $12 \mathrm{~h}$ until rats were sacrificed at $72 \mathrm{~h}$ after cortical injury (28). Thus, the dose and duration of progesterone used in the present study is able to produce neuroprotection to prevent the death of neurons.

Nogo-A, highly expressed in the myelin, has been regarded as the strongest inhibitor of axonal growth (7). Nogo-A has been extensively studied for its roles in inhibiting axonal regeneration in the injured CNS (31-33). The present study revealed that Nogo-A expression is increased at 1 day post-injury and is maintained at a high level for $\sim 4$ weeks, suggesting that the long-lasting presence of Nogo-A may contribute to the failure of neuronal regeneration following brain injury. It has been reported that the neutralization of Nogo-A with the anti-Nogo-A antibodies induces neuronal growth following brain injury $(33,34)$, suggesting that the inhibition of Nogo-A may be useful for the treatment of TBI. In agreement with this theory, the present study revealed that progesterone inhibits Nogo-A expression in TBI rats from 1-28 days post-injury, suggesting that progesterone can decrease the inhibitory effect of Nogo-A on neuronal regeneration. Therefore, our results suggest that progesterone may improve neuronal regeneration through the inhibition of Nogo-A. However, it remains unclear how progesterone inhibits Nogo-A expression in TBI.

GFAP, a specific marker for mature astrocytes, is important in maintaining the normal morphology and function of astrocytes in the CNS. Following brain injury, astrocytes begin to proliferate and increase the expression of GFAP $(12,15)$. Increased GFAP expression is a hallmark of reactive astrocytes, which is a major cellular component of the glial scar. The glial scar has long been implicated as a major barrier for axonal regeneration $(11,12)$. Inhibition of the glial scar is considered to be a therapeutic strategy for TBI (35). Therefore, the optimum time window for inhibiting glial scar formation is critical for therapy. The present study demonstrated that GFAP expression reaches a peak at 3 days post-injury and declines quickly to normal control levels at 28 days post-injury, suggesting that increased GFAP expression does not last long and glial scar formation starts early (1-3 days) following brain injury. In addition, our data demonstrated that progesterone inhibits GFAP expression at early time points (1-3 days) post-injury, suggesting that progesterone is a good drug to prevent glial scar formation. However, the exact mechanism underlying the effects of progesterone on glial scar formation remains to be determined.

GAP-43, the intracellular growth associated protein, is important in neuron growth during brain development and neuroregeneration following TBI (16-18). Following brain injury, GAP-43 protein synthesis greatly increases and is shuttled along the axon by fast axonal transport to the site of injury to promote axonal regeneration $(17,19,21,22)$. Consistent with these studies, the present study found that GAP-43 protein expression is increased following TBI. Progesterone treatment further increases the expression of GAP-43, suggesting that progesterone may promote axonal regeneration. However, progesterone has been reported to inhibit the high expression of GAP-43 mRNA in Wobbler mice, which exhibit motor neuron degeneration (36). Although increased expression of GAP-43 has been identified in a TBI rat model in the present study and in Wobbler mice, the mechanisms underlying the overexpression of GAP-43 remain unclear in these diseases. It has been reported that it is not the injury per se, but the disconnection of axons from peripheral target tissues regulating the expression of GAP-43 (37). It is also suggested that the disruption of the neuronal membrane due to the impact and shearing forces caused by the TBI may result in TBI-induced increases in the expression of GAP-43 (38). The neuronal degeneration in Wobbler mice caused by an autosomal recessive mutation is characterized by perikayal vascular degeneration and abnormalities of mitochondrial function in motor neurons $(25,39)$. The inhibition of GAP-43 expression by progesterone in Wobbler mice is proposed to be 
associated with the antioxidative effect of progesterone (40), while numerous cellular mechanisms have been reported to be involved in the neuroprotective effect of progesterone following TBI, including the reduction of edema, inflammation and apoptosis $(2-6,29,30)$. The difference in GAP-43 expression in response to progesterone treatment may largely result from the different pathogenesis of the diseases and the multiple mechanisms of action of progesterone.

In addition, the present study revealed that GAP-43 upregulation lasts $\sim 1$ week and its protein level gradually declines to a normal control level within 4 weeks post-injury, suggesting that GAP-43 promotes axonal regeneration at the early stage of brain injury, and its neuroregenerative effects are gradually reduced. Progesterone increases GAP-43 expression within 28 days post-injury, suggesting that progesterone is able to promote axonal regeneration following TBI through the upregulation of GAP-43. However, the time course of GAP-43 expression in the process of neuroregeneration following TBI has not been well established. It has been reported that the expression of GAP-43 is differentially regulated in CNS development and regeneration (41). The mechanisms underlying neuroregeneration following TBI and the beneficial effect of progesterone on the upregulation of GAP-43 need to be further determined.

The function of GAP-43 is dependent on PKC-mediated phosphorylation at S41. The regulation of GAP-43 at S41 has been reported to be associated with its distribution to different membrane domains, its regulation of actin filaments, its interaction with $\mathrm{Ca} /$ calmodulin and its binding to PIP2 $(17,42)$. In the present study, immunocytochemistry was performed to study the protein expression of GAP-43 following TBI, however, not the phosphorylation of GAP-43. It remains to be elucidated whether progesterone regulates the phosphorylation of GAP-43 at S41.

Preclinical studies have demonstrated that progesterone administered during the first few hours to days following injury significantly improved behavioral recovery in rats $(27,43,44)$. Two Phase II clinical trials have demonstrated that progesterone treatment has an improved survival and functional outcome than the placebo control in the treatment of TBI patients, indicating that progesterone is a safe and efficacious treatment for TBI $(45,46)$. A study using a rat model of TBI demonstrated that a continuous infusion of progesterone produces more behavioral recovery in TBI rats than bolus injections of progesterone (43). These results suggest that a continuous mode of progesterone administration may be more beneficial in the clinical testing than bolus injection in the treatment of TBI. In the present study, a bolus injection of progesterone was used to treat TBI and found that progesterone significantly inhibited the expression of Nogo-A and GFAP, and increased the GAP-43 expression. Although a continuous mode of progesterone administration was not used in the present, it is expected that a continuous mode of progesterone administration may produce more apparent effects on the expression of Nogo-A, GFAP and GAP-43.

In summary, the present study investigated the temporal changes of Nogo-A, GFAP and GAP-43 protein expression in TBI rats and the effects of progesterone on Nogo-A, GFAP and Gap-43 protein expression. Progesterone significantly inhibited the expression of Nogo-A and GFAP expression and increased the GAP-43 expression, suggesting that progesterone is able to promote neuroprotection following TBI.

\section{References}

1. Asemota AO, George BP, Bowman SM, Haider AH and Schneider EB: Causes and trends in traumatic brain injury for United States adolescents. J Neurotrauma 30: 67-75, 2013.

2. Feeser VR and Loria RM: Modulation of traumatic brain injury using progesterone and the role of glial cells on its neuroprotective actions. J Neuroimmunol 237: 4-12, 2011.

3. Gibson CL, Gray LJ, Bath PM and Murphy SP: Progesterone for the treatment of experimental brain injury; a systematic review. Brain 131: 318-328, 2008.

4. Roof RL, Duvdevani R, Braswell L and Stein DG: Progesterone facilitates cognitive recovery and reduces secondary neuronal loss caused by cortical contusion injury in male rats. Exp Neurol 129: 64-69, 1994.

5. Roof RL, Duvdevani R and Stein DG: Progesterone treatment attenuates brain edema following contusion injury in male and female rats. Restor Neurol Neurosci 4: 425-427, 1992.

6. Gibson CL, Constantin D, Prior MJ, Bath PM and Murphy SP: Progesterone suppresses the inflammatory response and nitric oxide synthase-2 expression following cerebral ischemia. Exp Neurol 193: 522-530, 2005.

7. Horner PJ and Gage FH: Regenerating the damaged central nervous system. Nature 407: 963-970, 2000.

8. Xiong NX, Zhao HY, Zhang FC and He ZQ: Negative correlation of Nogo-A with the malignancy of oligodendroglial tumor. Neurosci Bull 23: 41-45, 2007.

9. Hsieh SH, Ferraro GB and Fournier AE: Myelin-associated inhibitors regulate cofilin phosphorylation and neuronal inhibition through LIM kinase and Slingshot phosphatase. J Neurosci 26: 1006-1015, 2006.

10. Wang KC, Koprivica V, Kim JA, Sivasankaran R, Guo Y, Neve RL and He Z: Oligodendrocyte-myelin glycoprotein is a Nogo receptor ligand that inhibits neurite outgrowth. Nature 417: 941-944, 2002.

11. Yiu G and He Z: Glial inhibition of CNS axon regeneration. Nat Rev Neurosci 7: 617-627, 2006.

12. Fitch MT and Silver J: CNS injury, glial scars, and inflammation: Inhibitory extracellular matrices and regeneration failure. Exp Neurol 209: 294-301, 2008.

13. Brenneman MM, Wagner SJ, Cheatwood JL, Heldt SA, Corwin JV, Reep RL, Kartje GL, Mir AK and Schwab ME: Nogo-A inhibition induces recovery from neglect in rats. Behav Brain Res 187: 262-272, 2008.

14. Wang H, Yao YJ and Chen DP: Expression of Nogo-A mRNA and Nogo-A protein in brain tissue of neonatal mice with ischemic-hypoxic brain damage. Zhonghua Er Ke Za Zhi 44: 792-793, 2006 (In Chinese).

15. Stichel CC and Müller HW: The CNS lesion scar: new vistas on an old regeneration barrier. Cell Tissue Res 294: 1-9, 1998.

16. Clayton GH, Mahalik TJ and Finger TE: Expression of GAP43 mRNA in normally developing and transplanted neurons from the rat ventral mesencephalon. J Comp Neurol 347: 470-480, 1994.

17. Denny JB: Molecular mechanisms, biological actions, and neuropharmacology of the growth-associated protein GAP-43. Curr Neuropharmacol 4: 293-304, 2006.

18. Benowitz LI and Routtenberg A: GAP-43: an intrinsic determinant of neuronal development and plasticity. Trends Neurosci 20: 84-91, 1997.

19. Donovan SL and McCasland JS: GAP-43 is critical for normal targeting of thalamocortical and corticothalamic, but not trigeminothalamic axons in the whisker barrel system. Somatosens Mot Res 25: 33-47, 2008.

20. Hughes-Davis EJ, Cogen JP, Jakowec MW, Cheng HW, Grenningloh G, Meshul CK and McNeill TH: Differential regulation of the growth-associated proteins GAP-43 and superior cervical ganglion 10 in response to lesions of the cortex and substantia nigra in the adult rat. Neuroscience 135: 1231-1239, 2005.

21. Nagamoto-Combs K, Morecraft RJ, Darling WG and Combs CK: Long-term gliosis and molecular changes in the cervical spinal cord of the rhesus monkey after traumatic brain injury. J Neurotrauma 27: 565-585, 2010. 
22. Schmidt-Kastner R, Bedard A and Hakim A: Transient expression of GAP-43 within the hippocampus after global brain ischemia in rat. Cell Tissue Res 288: 225-238, 1997.

23. Labombarda F, González Deniselle MC, De Nicola AF and González SL: Progesterone and the spinal cord: good friends in bad times. Neuroimmunomodulation 17: 146-149, 2010.

24. Garay L, Deniselle MC, Meyer M, Costa JJ, Lima A, Roig P and De nicola AF: Protective effects of progesterone administration on axonal pathology in mice with experimental autoimmune encephalomyelitis. Brain Res 1283: 177-185, 2009.

25. De Nicola AF, Labombarda F, Deniselle MC, Gonzalez SL, Garay L, Meyer M, Gargiulo G, Guennoun R and Schumacher M: Progesterone neuroprotection in traumatic CNS injury and motoneuron degeneration. Front Neuroendocrinol 30: 173-187, 2009.

26. Feeney DM, Boyeson MG, Linn RT, Murray HM and Dail WG: Responses to cortical injury: I. Methodology and local effects of contusions in the rat. Brain Res 211: 67-77, 1981

27. Goss CW, Hoffman SW and Stein DG: Behavioral effects and anatomic correlates after brain injury: a progesterone dose-response study. Pharmacol Biochem Behav 76: 231-242, 2003.

28. Anderson GD, Farin FM, Bammler TK, Beyer RP, Swan AA, Wilkerson HW, Kantor ED and Hoane MR: The effect of progesterone dose on gene expression after traumatic brain injury. J Neurotrauma 28: 1827-1843, 2011.

29. Pajović SB, Saicić ZS, Spasić MB, Petrović VM and Martinović JV: Effects of progesterone and estradiol benzoate on glutathione dependent antioxidant enzyme activities in the brain of female rats. Gen Physiol Biophys 18: 35-44, 1999.

30. Pierson RC, Lyons AM and Greenfield LJ, Jr: Gonadal steroids regulate GABAA receptor subunit mRNA expression in NT2-N neurons. Brain Res Mol Brain Res 138: 105-115, 2005.

31. GrandPré T, Nakamura F, Vartanian T and Strittmatter SM: Identification of the Nogo inhibitor of axon regeneration as a Reticulon protein. Nature 403: 439-444, 2000.

32. Oertle T, van der Haar ME, Bandtlow CE, et al: Nogo-A inhibits neurite outgrowth and cell spreading with three discrete regions. J Neurosci 23: 5393-5406, 2003.

33. Marklund N, Bareyre FM, Royo NC, Thompson HJ, Mir AK, Grady MS, Schwab ME and McIntosh TK: Cognitive outcome following brain injury and treatment with an inhibitor of Nogo-A in association with an attenuated downregulation of hippocampal growth-associated protein-43 expression. J Neurosurg 107: 844-853, 2007.

34. Lenzlinger PM, Shimizu S, Marklund N, et al: Delayed inhibition of Nogo-A does not alter injury-induced axonal sprouting but enhances recovery of cognitive function following experimental traumatic brain injury in rats. Neuroscience 134: 1047-1056, 2005
35. Mueller BK, Mueller R and Schoemaker H: Stimulating neuroregeneration as a therapeutic drug approach for traumatic brain injury. Br J Pharmacol 157: 675-685, 2009.

36. Gonzalez Deniselle MC, Lopez Costa JJ, Gonzalez SL, Labombarda F, Garay L, Guennoun R, Schumacher M and De Nicola AF: Basis of progesterone protection in spinal cord neurodegeneration. J Steroid Biochem Mol Biol 83: 199-209, 2002.

37. Schreyer DJ and Skene JH: Injury-associated induction of GAP-43 expression displays axon branch specificity in rat dorsal root ganglion neurons. J Neurobiol 24: 959-970, 1993.

38. Hulsebosch CE, DeWitt DS, Jenkins LW and Prough DS: Traumatic brain injury in rats results in increased expression of Gap-43 that correlates with behavioral recovery. Neurosci Lett 255: 83-86, 1998.

39. Santoro B, Bigini P, Levandis G, Nobile V, Biggiogera M, Botti F, Mennini T and Curti D: Evidence for chronic mitochondrial impairment in the cervical spinal cord of a murine model of motor neuron disease. Neurobiol Dis 17: 349-357, 2004.

40. González Deniselle MC, González SL and De Nicola AF: Cellular basis of steroid neuroprotection in the wobbler mouse, a genetic model of motoneuron disease. Cell Mol Neurobiol 21: $237-254,2001$

41. Udvadia AJ, Köster RW and Skene JH: GAP-43 promoter elements in transgenic zebrafish reveal a difference in signals for axon growth during CNS development and regeneration. Development 128: 1175-1182, 2001.

42. Nguyen L, He Q and Meiri KF: Regulation of GAP-43 at serine 41 acts as a switch to modulate both intrinsic and extrinsic behaviors of growing neurons, via altered membrane distribution. Mol Cell Neurosci 41: 62-73, 2009.

43. Cutler SM, VanLandingham JW, Murphy AZ and Stein DG: Slow-release and injected progesterone treatments enhance acute recovery after traumatic brain injury. Pharmacol Biochem Behav 84: 420-428, 2006

44. Wali B, Sayeed I and Stein DG: Improved behavioral outcomes after progesterone administration in aged male rats with traumatic brain injury. Restor Neurol Neurosci 29: 61-71, 2011.

45. Wright DW, Kellermann AL, Hertzberg VS, et al: ProTECT: a randomized clinical trial of progesterone for acute traumatic brain injury. Ann Emerg Med 49: 391-402, 2007.

46. Xiao G, Wei J, Yan W, Wang W and Lu Z: Improved outcomes from the administration of progesterone for patients with acute severe traumatic brain injury: a randomized controlled trial. Crit Care 12: R61, 2008. 